\title{
Théorème local pour chaînes de Markov de probabilité de transition quasi-compacte. Applications aux chaînes $V$-géométriquement ergodiques et aux modèles itératifs
}

\author{
Loïc Hervé \\ I.R.M.A.R., UMR-CNRS 6625, Institut national des sciences appliquées de Rennes, 20, avenue des Buttes de Couësmes CS 14315 , \\ 35043 Rennes Cedex, France
}

Reçu le 22 octobre 2003 ; reçu en forme révisée le 23 avril 2004 ; accepté le 4 mai 2004

Disponible sur Internet le 28 septembre 2004

\begin{abstract}
Résumé
Soit $Q$ une probabilité de transition sur un espace mesurable $E$, soit $\left(X_{n}\right)_{n}$ une chaîne de Markov associée à $Q$, et enfin soit $\xi$ une fonction réelle mesurable sur $E$ telle que $\left(\xi\left(X_{n}\right)\right)_{n} \geqslant 0$ vérifie un théorème limite central. Sous des hypothèses fonctionnelles sur l'action de $Q$ et de ses noyaux de Fourier $Q(t)$, nous démontrons dans ce papier un théorème limite local. Nous utilisons la méthode spectrale de Nagaev améliorée par un théorème de perturbation de Keller et Liverani. Les conditions requises dans ce travail sur $Q(t)$ sont plus faibles que celles habituellement imposées lorsqu'on utilise le théorème de perturbation standard. Nous présentons des applications aux chaînes $V$-géométriquement ergodiques et aux modèles itératifs Lipschitziens.
\end{abstract}

(c) 2004 Elsevier SAS. Tous droits réservés.

\begin{abstract}
Let $Q$ be a transition probability on a measurable space $E$, let $\left(X_{n}\right)_{n}$ be a Markov chain associated to $Q$, and let $\xi$ be a realvalued measurable function on $E$ such that $\left(\xi\left(X_{n}\right)\right)_{n \geqslant 0}$ satisfies a central limit theorem. Under functional hypotheses on the action of $Q$ and its Fourier kernels $Q(t)$, we establish a local limit theorem. We use the spectral method of Nagaev improved by a perturbation theorem of Keller and Liverani. The conditions required here on $Q(t)$ are weaker than the ones usually imposed when the standard perturbation theorem is used in the spectral method. We give some applications to $V$-geometric ergodic chains and to Lipschitz iterative models.
\end{abstract}

(C) 2004 Elsevier SAS. Tous droits réservés.

Adresse e-mail : loic.herve@insa-rennes.fr (L. Hervé). 


\section{Introduction}

Soit $(E, \mathcal{E})$ un espace mesurable, soit $Q$ une probabilité de transition sur $(E, \mathcal{E})$, et soit $\left(X_{n}\right)_{n} \geqslant 0$ une chaîne de Markov sur $(E, \mathcal{E})$ associée à $Q$. On désigne par $\xi$ une fonction mesurable sur $E$, à valeurs dans $\mathbb{R}$, et l'on pose $S_{n}=\sum_{k=1}^{n} \xi\left(X_{k}\right)$.

Dans toute la suite, nous supposerons qu'il existe sur $E$ une probabilité $Q$-invariante, notée $v$, que $\xi$ est $\nu$-intégrable, et enfin que $v(\xi)=0$ (quitte à remplacer $\xi$ par $\xi-v(\xi)$, on peut toujours se ramener à cette condition).

L'objet principal de ce travail est d'établir, sous des hypothèses précisées au paragraphe 2, un théorème limite local pour la suite de variables aléatoires $\left(\xi\left(X_{n}\right)\right)_{n} \geqslant 0$. Ce théorème a été initialement obtenu par Kolmogorov [17] dans le cadre des chaînes de Markov finies. ${ }^{1}$ Grâce à des techniques de perturbation d'opérateurs, un théorème limite local fut ensuite établi par Nagaev [20,21] sous une hypothèse de quasi-compacité (cf. (F2) § 2) du noyau $Q$. La méthode spectrale de Nagaev a été par la suite largement appliquée dans le but d'obtenir non seulement un théorème local, mais également un théorème limite central avec vitesse, un théorème de renouvellement, et des théorèmes de grandes déviations. Pour le théorème local, citons par exemple $[7,26]$, et $[24,5,23]$ dans le cadre de systèmes dynamiques.

On trouvera une description générale de la méthode spectrale dans [12]. Rappelons que, en substance, celle-ci peut être décomposée selon les quatre étapes (a)-(d) qui suivent :

(a) Faire le lien entre la fonction caractéristique de $S_{n}$ et les itérés des noyaux de Fourier $Q(t)=\mathrm{e}^{i t \xi} Q$ associés à $Q$ et $\xi$ (voir la formule (2) $d u \S 4.3$ ).

(b) À l'aide d'un théorème de perturbation d'opérateurs appliqué à $Q(\cdot)$, établir que, pour $|t|$ assez petit, $Q(t)$ vérifie les mêmes propriétés spectrales que $Q$; en particulier $Q(t)$ aura une valeur propre dominante $\lambda(t)$ proche de $\lambda=1$ en $t=0$.

(c) À l'aide d'hypothèses de régularité sur $Q(\cdot)$, obtenir des précisions sur le comportement en $t=0$ des éléments perturbés, notamment de $\lambda(t)$.

(d) Appliquer ensuite, comme dans le cas des suites de v.a.i.i.d, les techniques usuelles de transformée de Fourier.

Les difficultés résident ici dans les étapes (b) et (c). Dans les travaux cités ci-dessus, celles-ci sont traitées parallèlement en appliquant à $Q(\cdot)$, dans son action sur un certain espace $\mathcal{B}$, le théorème standard de perturbation d'opérateurs : il suffit pour cela que l'application $Q(\cdot)$, à valeurs dans l'espace des endomorphismes continus de $\mathcal{B}$, soit suffisamment régulière. Cette technique est souvent bien adaptée lorsque la fonction $\xi$ est bornée sur $E .^{2}$ Cette dernière condition est d'ailleurs imposée dans tous les travaux mentionnés plus haut (excepté [12]).

Malheureusement, si $\xi$ est non bornée, l'application du théorème standard de perturbation d'opérateurs requiert des hypothèses assez contraignantes sur $Q$, par exemple des conditions de moments exponentiels dans le cadre des noyaux Lipschitziens, voir [19] et [12] (Chap. X).

Dans ce même contexte d'itérés de transformations Lipschitziennes, une première amélioration a été apportée dans [13] où l'on établit, sous des hypothèses de moments polynomiaux, un théorème limite local et un théorème limite central avec vitesse. Les idées dans [13] sont

- Pour l'étape (b) : remplacer le théorème classique de perturbation d'opérateurs par un résultat de KellerLiverani [16]. ${ }^{3}$

\footnotetext{
1 À notre connaissance la méthode de [17] n'a pas été généralisée.

2 En fait le théorème standard de perturbation d'opérateurs s'applique aisément à $Q(t)$ lorsque $\mathcal{B}$ est une algèbre de Banach et que $\xi \in \mathcal{B} ;$ en effet, dans ce cas, $Q(\cdot)$ est analytique (voir par ex. [12, Lemme VIII.10]).

${ }^{3}$ Le théorème de [16] est particulièrement adapté lorsque $Q(t)$ vérifie les conditions du théorème de Ionescu-Tulcea et Marinescu [15] (en substance les conditions (F3) (F5) du § 2).
} 
- Pour l'étape (c) : considérer $Q(t)$, non pas comme un endomorphisme de $\mathcal{B}$, mais comme une application linéaire entre deux espaces convenablement choisis.

Dans ce papier, le cadre est plus général qu'en [13], mais nous n'abordons pas la question de vitesse dans le théorème limite central. Nous nous intéressons principalement au théorème limite local, et notre méthode peut être résumée comme suit.

- Pour l'étape (b) : grâce aux hypothèses fonctionnelles $(\mathcal{F})$ du $\S 2$, nous appliquerons à nouveau aux noyaux $Q(t)$ le théorème de Keller-Liverani (cf. Th. (K-L), § 4).

- Pour l'étape (c) : l'étude du comportement en $t=0$ de la valeur propre perturbée $\lambda(t)$ est fondée, non plus sur la régularité de $Q(\cdot)$, mais sur un argument de nature probabiliste : plus précisément nous supposons que $\left(\xi\left(X_{n}\right)\right)_{n \geqslant 0}$ vérifie un théorème limite central (t.l.c.) dans le cas stationnaire, et nous démontrons que cette hypothèse implique pour $\lambda(t)$ un développement limité à l'ordre 2 en $t=0$ (cf. Lemme 4.2).

Les énoncés, présentés au paragraphe 2, portent plus généralement sur la suite de v.a $\left(X_{n}, S_{n}\right)_{n}$. Ils contiennent un théorème limite central et un théorème limite local, dans les cas stationnaire et non stationnaire. Quelques remarques relatives aux hypothèses sont regroupées à la fin du paragraphe 2. Nous présentons au paragraphe 3 des applications et exemples, notamment dans le cadre des chaînes $V$-géométriquement ergodiques et des modèles itératifs. Le paragraphe 4 est consacré aux preuves des théorèmes limites.

Notations. Si $X$ et $Y$ sont des espaces de Banach, nous désignons par $X^{\prime}$ le dual topologique de $X$, par $\mathcal{L}(X)$ l'espace des endomorphismes continus de $X$, et $\operatorname{par} \mathcal{L}(X, Y)$ l'espace des applications linéaires continues de $X$ dans $Y$. Ces espaces sont munis des normes subordonnées. On note $\langle\cdot, \cdot\rangle$ le crochet de dualité sur $X^{\prime} \times X$. Si $T \in \mathcal{L}(X), r(T)$ désigne le rayon spectral de $T$, et $T^{*}$ l'opérateur adjoint de $T$.

Rappelons que $v$ désigne une probabilité $Q$-invariante. Pour $p \geqslant 1$ on note $\mathbb{L}^{p}(v)$ l'espace de Lebesgue usuel associé à $v$. On note $\mathbf{1}=1_{E}$ la fonction identiquement égale à 1 sur $E$.

Si $\mu$ est une probabilité sur $E$, nous noterons parfois $\mu(f)=\int_{E} f d \mu$. Si $X$ est composé de fonctions mesurables sur $E$, à valeurs complexes, et si toute fonction $f \in X$ est $\mu$-intégrable, $\mu$ s'identifie ainsi à une forme linéaire sur $X$; nous écrirons $\mu \in X^{\prime}$ lorsque celle-ci est continue.

La probabilité initiale de la chaîne, c'est-à-dire la loi de $X_{0}$, sera appelée $\mu_{0}$.

$L$ est la mesure de Lebesgue sur $\mathbb{R}$, et $\mathcal{N}\left(0, \sigma^{2}\right)$ est la loi normale centrée, de variance $\sigma^{2}>0$.

Enfin les noyaux de Fourier associés à $Q$ et $\xi$ sont définis par

$$
Q(t)(x, d y)=\mathrm{e}^{i t \xi} Q(x, d y), \quad t \in \mathbb{R}, x \in E .
$$

\section{Hypothèses et énoncés des résultats}

Hypothèse $(\mathcal{P})$. Sous la probabilité $\mathbb{P}_{v},\left(\frac{S_{n}}{\sqrt{n}}\right)_{n}$ converge en loi vers $\mathcal{N}\left(0, \sigma^{2}\right)\left(\sigma^{2}>0\right)$.

Dans toute la suite, nous désignons par $(\mathcal{B},\|\cdot\|)$ un espace de Banach composé de fonctions (ou de classes de fonctions) mesurables sur $E$, à valeurs complexes, et nous supposons que $1 \in \mathcal{B}, \mathcal{B}$ est réticulé (i.e. $f \in \mathcal{B} \Rightarrow$ $|f| \in \mathcal{B})$, et $Q \in \mathcal{L}(\mathcal{B}){ }^{4}$

Nous ferons appel aux hypothèses fonctionnelles suivantes.

\footnotetext{
$\overline{4}$ I.e. Pour tous $f \in \mathcal{B}$ et $x \in E, f$ est $Q(x, \cdot)$-intégrable, $Q f=\int_{E} f(y) Q(\cdot, d y) \in \mathcal{B}$, et cette formule définit un endomorphisme continu sur $\mathcal{B}$.
} 
Hypothèse $(\mathcal{F})$. Les conditions $(\mathrm{F} 1)-(\mathrm{F} 5)$ ci-dessous sont satisfaites :

(F1) Il existe $q \in] 1,+\infty]$ tel que $\mathcal{B}$ s'envoie continûment dans $\mathbb{L}^{q}(v) .^{5}$

(F2) Il existe $\kappa_{0}<1$ et $C \geqslant 0$ tels que $: \forall n \geqslant 1, \forall f \in \mathcal{B},\left\|Q^{n} f-v(f)\right\| \leqslant C \kappa_{0}^{n}\|f\| .^{6}$

(F3) $Q(\{f \in \mathcal{B},\|f\| \leqslant 1\})$ est relativement compacte dans $(\mathcal{B}, v(|\cdot|)){ }^{7}$

(F4) Pour tout $t \in \mathbb{R}, f \mapsto \mathrm{e}^{i t \xi} f$ définit un endomorphisme continu sur $\mathcal{B} .8,9$

(F5) Il existe un intervalle ouvert I contenant $t=0$, puis $N \in \mathbb{N}^{*}, \kappa<1, C \geqslant 0$ tels que

$$
\forall t \in I, \forall f \in \mathcal{B}, \quad\left\|Q(t)^{N} f\right\| \leqslant \kappa^{N}\|f\|+C v(|f|) \cdot{ }^{10}
$$

Hypothèse ( $\xi$ est non-arithmétique). Pour tous réels $0<\alpha<\beta$, il existe $\rho<1, c \geqslant 0$ tels que l'on ait

$$
\forall n \geqslant 1, \quad \sup _{\alpha \leqslant|t| \leqslant \beta}\left\|Q(t)^{n}\right\| \leqslant c \rho^{n} .1^{11}
$$

Hypothèse $(\widetilde{\mathcal{F}})$. Le couple $(Q, \mathcal{B})$ vérifie $(\mathcal{F})$, et il existe un espace de Banach $\widetilde{\mathcal{B}}$ tel que :

- $\mathcal{B}$ s'envoie continûment dans $\widetilde{\mathcal{B}}$,

$-v \in \widetilde{\mathcal{B}}^{\prime}$,

- $Q \in \mathcal{L}(\widetilde{\mathcal{B}})$ et $(Q, \widetilde{\mathcal{B}})$ vérifie $(\mathrm{F} 2)$,

- Quand $t \rightarrow 0, Q(t)$ converge vers $Q$ dans $\mathcal{L}(\mathcal{B}, \widetilde{\mathcal{B}})$.

Dans le théorème ci-dessous, on s'intéresse aux deux propriétés suivantes :

(TLC) Pour tout $f \in \mathcal{B}, f \geqslant 0$, et pour toute fonction réelle g continue bornée sur $\mathbb{R}$,

$$
\lim _{n} \mathbb{E}_{\mu_{0}}\left[f\left(X_{n}\right) g\left(\frac{S_{n}}{\sqrt{n}}\right)\right]=v(f) \mathcal{N}\left(0, \sigma^{2}\right)(g)
$$

(TLL) Pour tout $f \in \mathcal{B}, f \geqslant 0$, et toute fonction réelle $g$ continue à support compact sur $\mathbb{R}$,

$$
\limsup _{n}\left|\sigma \sqrt{2 \pi n} \mathbb{E}_{\mu_{0}}\left[f\left(X_{n}\right) g\left(S_{n}-u\right)\right]-\mathrm{e}^{\frac{-u^{2}}{2 n \sigma^{2}}} v(f) L(g)\right|=0 .
$$

\section{Théorème 2.1.}

CAS STATIONNAIRE. Supposons que $\mu_{0}=v$. Alors, sous les hypothèses $(\mathcal{P})$ et $(\mathcal{F})$, la propriété $(\mathrm{TLC})$ est satisfaite. Si en outre $\xi$ est non-arithmétique, alors la propriété (TLL) est vérifiée.

CAS NON STATIONNAIRE. Supposons que les hypothèses $(\mathcal{P})$ et $(\widetilde{\mathcal{F}})$ soient satisfaites, et que $\mu_{0} \in \widetilde{\mathcal{B}}^{\prime}$. Alors la propriété (TLC) est satisfaite. Si en outre $\xi$ est non-arithmétique, la propriété (TLL) est vérifiée.

\footnotetext{
$\overline{5}$ I.e. $\exists A>0, \forall f \in \mathcal{B},\left(v\left(|f|^{q}\right)^{1 / q} \leqslant A\|f\|\right.$. Cette condition implique que $v \in \mathcal{B}^{\prime}$, cf. Rq. 2.

${ }^{6}$ I.e. $Q$ est quasi-compact à itérés bornés sur $\mathcal{B}$, avec 1 comme valeur propre simple et unique valeur propre périphérique, voir Rq. 3.

7 I.e. $\mathrm{Si}\left(f_{n}\right)_{n} \in \mathcal{B}^{\mathbb{N}},\left\|f_{n}\right\| \leqslant 1$, alors il existe une sous-suite $\left(n_{k}\right)_{k}$ et $g \in \mathcal{B}$ tels que $\lim _{k} v\left(\left|Q f_{n_{k}}-g\right|\right)=0$.

8 I.e. $\forall t \in \mathbb{R}, \forall f \in \mathcal{B}, \mathrm{e}^{i t \xi} f \in \mathcal{B}$, et $\forall t \in \mathbb{R}, \exists M_{t}>0, \forall f \in \mathcal{B},\left\|\mathrm{e}^{i t \xi} f\right\| \leqslant M_{t}\|f\|$.

${ }^{9}$ Les conditions (F3)-(F4) peuvent être remplacées par : pour $t \in \mathbb{R}, Q(t) \in \mathcal{L}(\mathcal{B})$ et pour $|t|$ petit, $Q(t)(\{f \in \mathcal{B},\|f\| \leqslant 1\})$ est relativement compacte dans $(\mathcal{B}, v(|\cdot|))$, cf. Rq. 4

10 I.e. Les inégalités de type (F5) sont dites de Doeblin-Fortet; elles interviennent par exemple dans le théorème de Ionescu-Tulcea et Marinescu. Voir Rq. 3.

11 Il est sous-entendu ici que les noyaux $Q(t)$ ont une action continue sur $\mathcal{B}$. Sous des conditions assez générales, un critère simple de non-arithméticité sera donné dans la Remarque 6.
} 
Le cas $f=\mathbf{1}$ dans (TLC) [resp. $f=\mathbf{1}$ et $u=0$ dans (TLL)] correspond pour la suite $\left(\xi\left(X_{n}\right)\right)_{n} \geqslant 0$ à l'énoncé standard du théorème limite central [resp. du théorème limite local]. Évidemment, pour $f=\mathbf{1}$ et $\mu_{0}=v$, la propriété (TLC) coïncide avec l'hypothèse $(\mathcal{P})$.

Remarque 1 (Une condition suffisante pour $(\mathcal{P})$ ). Dans le cadre des chaînes de Markov, plusieurs méthodes existent, notamment dans le cas stationnaire, pour établir le t.l.c. La plus naturelle dans ce papier, du fait de l'hypothèse (F2), est la méthode de Gordin. On dispose par exemple du résultat suivant. Supposons que $\left(X_{n}\right)_{n \geqslant 0}$ soit la chaîne de Markov canonique associée à $Q$, et que la sous-tribu de $\mathcal{E}$ engendrée par les fonctions de $\mathcal{B}$ coïncide avec $\mathcal{E}$.

Proposition 2.2. Si $\mathcal{B}$ s'envoie continûment dans $\mathbb{L}^{2}(v)$ (i.e. $(\mathrm{F} 1)$ avec $\left.q=2\right)$, si $\xi \in \mathcal{B}$, et enfin si $(Q, \mathcal{B})$ vérifie la condition $(\mathrm{F} 2)$, alors la suite de v.a. $\left(\frac{S_{n}}{\sqrt{n}}\right)_{n}$ converge en loi, sous la probabilité $\mathbb{P}_{v}$, vers la gaussienne $\mathcal{N}\left(0, \sigma^{2}\right)$, avec $\sigma^{2}=v\left(\tilde{\xi}^{2}\right)-v\left((Q \tilde{\xi})^{2}\right)$, où l'on a noté $\tilde{\xi}=\sum_{n \geqslant 0} Q^{n} \xi$ (le cas $\sigma^{2}=0$ n'est pas exclu dans cet énoncé).

Preuve. On suit ici la méthode de Gordin [8,9]. La série $\sum_{n \geqslant 0} Q^{n} \xi$ converge absolument dans $\mathcal{B}$, et sa somme $\tilde{\xi}$ vérifie l'équation de Poisson $\tilde{\xi}-Q \tilde{\xi}=\xi$. Soit $Y_{k}=\tilde{\xi}\left(X_{k}\right)-Q \tilde{\xi}\left(X_{k-1}\right)$. Alors $\left(Y_{k}\right)_{k}$ est une suite d'accroissements de martingales, en outre $Y_{k}$ est de carré $\mathbb{P}_{v}$-intégrable car $\tilde{\xi} \in \mathcal{B} \subset \mathbb{L}^{2}(v)$. En posant $Z_{n}=\tilde{\xi}\left(X_{1}\right)-Q \tilde{\xi}\left(X_{n}\right)$, l'équation de Poisson permet d'écrire $S_{n}=\sum_{k=2}^{n} Y_{k}+Z_{n}$. Par ailleurs, la condition (F2), et le fait que la tribu engendrée par $\mathcal{B}$ est par hypothèse égale à $\mathcal{E}$, entraînent que, sous la probabilité $\mathbb{P}_{v}$, la chaîne stationnaire $\left(X_{n}\right)_{n} \geqslant 0$ est ergodique (voir par exemple [12, Chap. IX]). Il vient que $\left(Y_{k}\right)_{k}$ est stationnaire et ergodique, par conséquent elle vérifie le t.l.c de Billingsley-Ibragimov $[3,14]$ avec $\sigma^{2}$ défini comme dans l'énoncé. Enfin, la suite $\left(\mathbb{E}_{\nu}\left[\left|Z_{n}\right|\right]\right)_{n}$ est bornée, donc $\left(\frac{Z_{n}}{\sqrt{n}}\right)_{n}$ converge en moyenne vers 0 . D'où le résultat de l'énoncé.

Remarque 2. L'hypothèse $(\mathrm{F} 1)$ servira à établir que $Q(\cdot)$ vérifie, vis-à-vis de la semi-norme $v(|\cdot|)$, une propriété très faible de continuité en $t=0$ (cf. (1) § 4.1) qui, adjointe aux hypothèses (F2) (F3) (F4) (F5), permettra d'appliquer les résultats de [16]. Observons également que (F1) implique $v \in \mathcal{B}^{\prime}$. En effet, pour $f \in \mathcal{B}$, on a $v(|f|) \leqslant\left[v\left(|f|^{q}\right)\right]^{1 / q} \leqslant A\|f\|$.

Remarque 3 (Une condition suffisante pour (F2)). La condition (F2) exprime le fait que $Q$ est quasi-compact à itérés bornés sur $\mathcal{B}$, et que 1 est valeur propre simple et l'unique valeur propre périphérique de $Q$. L'énoncé suivant est une conséquence du théorème de Ionescu-Tulcea et Marinescu [15], appliqué avec la semi-norme auxiliaire $v(|\cdot|)$.

Proposition 2.3. Si $Q$ vérifie (F3) et s'il existe $N \in \mathbb{N}^{*}, \kappa<1$ et $C \geqslant 0$ tels que

(A) $\forall f \in \mathcal{B}, \quad\left\|Q^{N} f\right\| \leqslant \kappa^{N}\|f\|+C v(|f|)$,

alors $Q$ est quasi-compact à itérés bornés sur $\mathcal{B}$. Si en outre 1 est valeur propre simple et l'unique valeur propre périphérique de $Q$, alors (F2) est vérifiée.

Preuve. Pour $n \geqslant 1$, on a, par invariance de $v, v\left(\left|Q^{n} f\right|\right) \leqslant v\left(Q^{n}|f|\right)=v(|f|)$. Grâce à cette remarque et aux conditions $(\mathrm{A})(\mathrm{F} 3)$, on déduit de [15] que $Q$ est quasi-compact à itérés bornés sur $\mathcal{B}$. La dernière assertion est évidente.

Notons que la condition (A) correspond au cas $t=0$ dans (F5). Par conséquent les conditions (F3) (F5) entrâ1nent que $Q$ est quasi-compact à itérés bornés sur $\mathcal{B}$. Les conditions ci-dessus sur la valeur propre 1 découlent le plus souvent d'hypothèses d'apériodicité et d'irréductibilité de la chaîne. 
Remarque 4 (Une condition plus générale pour $(\mathrm{F} 3)-(\mathrm{F} 4))$. On a $Q(t) f=Q\left(\mathrm{e}^{i t \xi} f\right)$. Ainsi, par composition, sous la condition (F4), on a $Q(t) \in \mathcal{L}(\mathcal{B})$. De même, par composition, les conditions (F3) (F4) impliquent que l'image par $Q(t)$ de la boule unité de $(\mathcal{B},\|\cdot\|)$ est relativement compacte dans $(\mathcal{B}, v(|\cdot|))$.

En fait, pour la validité du théorème de Keller-Liverani au § 4.1, les hypothèses (F3)-(F4) peuvent être remplacées par la condition suivante plus faible:

Condition $\left(\mathrm{F}_{3,4}\right)$. Pour $t \in \mathbb{R}$, on a $Q(t) \in \mathcal{L}(\mathcal{B})$, et pour $t \in I$, où $I$ est un intervalle ouvert contenant $t=0$, $Q(t)(\{f \in \mathcal{B},\|f\| \leqslant 1\})$ est relativement compacte dans $(\mathcal{B}, v(|\cdot|))$.

Nous obtenons ainsi un énoncé un peu plus général que le Théorème 2.1, à savoir :

Théorème $2.1^{\prime}$. Les conclusions du Théorème 2.1 subsistent lorsque $(\mathcal{F})$ et $(\widetilde{\mathcal{F}})$ sont remplacées respectivement par:

$\left(\mathcal{F}^{\prime}\right)(\mathrm{F} 1)(\mathrm{F} 2)\left(\mathrm{F}_{3,4}\right)(\mathrm{F} 5)$,

$\left(\widetilde{\mathcal{F}}^{\prime}\right)$ obtenue comme $(\widetilde{\mathcal{F}})$, avec $\left(\mathcal{F}^{\prime}\right)$ à la place de $(\mathcal{F})$.

Remarque 5 (Une condition suffisante simple pour (F5) dans un cas particulier). Les inégalités (F5), dites de Doeblin-Fortet, font partie des hypothèses du théorème de Keller-Liverani (§ 4.1). La propriété suivante sera utile au paragraphe 3.1 : les conditions (F2) et

$$
\text { pour } f, g \in \mathcal{B} \text { quelconques, } \quad|f| \leqslant|g| \Rightarrow\|f\|=\||f|\| \leqslant\||g|\|=\|g\|^{12}
$$

impliquent clairement (F4), puis (F5) avec $I=\mathbb{R}$.

En effet, de $\left|Q(t)^{n} f\right| \leqslant Q^{n}|f|$ et (F2), on déduit $\left\|Q(t)^{n} f\right\| \leqslant\left\|Q^{n}|f|\right\| \leqslant C \kappa_{0}^{n}\|f\|+v(|f|)\|\mathbf{1}\|$.

Remarque 6 (Un critère de non-arithméticité). Rappelons que $A \in \mathcal{E}$ est dite $Q$-invariante si $Q(a, A)=1$ pour tout $a \in A$. Supposons que, pour tout $x \in E$, l'application $f \mapsto f(x)$ soit dans $\mathcal{B}^{\prime}$, et que le produit de deux fonctions bornées de $\mathcal{B}$ appartienne à $\mathcal{B}$. Alors

Proposition 2.4. Sous l'hypothèse $(\mathcal{F})$ [ou bien $\left.\left(\mathcal{F}^{\prime}\right)\right]$, avec $I=\mathbb{R}$ dans $(\mathrm{F} 5)$ [et $\left.\left(F_{3,4}\right)\right]$, la fonction $\xi$ est nonarithmétique s'il n'existe pas de réels $t \neq 0$, ni $A \in \mathcal{E} Q$-invariant, ni $\lambda \in \mathbb{C},|\lambda|=1$, ni $w \in \mathcal{B}$ de module constant non nul sur A, tels que :

$$
\forall x \in A, \forall n \geqslant 1, \quad \exp \left(i t\left[\xi\left(x_{1}\right)+\cdots+\xi\left(x_{n}\right)\right]\right) w\left(x_{n}\right)=\lambda^{n} w\left(x_{0}\right) \quad \prod_{i=1}^{n} Q\left(x_{i-1}, \text { dxi }\right)-p . s .
$$

La preuve de cette proposition, dont une partie s'appuie sur le théorème de Keller-Liverani, est reportée au paragraphe 4.2.

Remarque 7. Si $\widetilde{\mathcal{B}}=\mathcal{B}$, l'hypothèse $(\widetilde{\mathcal{F}})$ se réduit à $:(Q, \mathcal{B})$ vérifie $(\mathcal{F})$ et, quand $t \rightarrow 0, Q(t)$ converge vers $Q$ dans $\mathcal{L}(\mathcal{B})$. Comme le montreront les exemples, cette dernière condition n'est pas adaptée en général lorsque $\xi$ est non bornée; elle l'est en revanche si l'on choisit convenablement un espace $\widetilde{\mathcal{B}}$ contenant strictement $\mathcal{B}$.

\footnotetext{
12 Un tel espace est appelé un treillis de Banach [25].
} 


\section{Applications et exemples}

\subsection{Application aux chaînes $V$-géométriquement ergodiques}

On suppose ici que $(E, d)$ est un espace métrique non compact tel que toute boule fermée de $E$ soit compacte. On munit $E$ de sa tribu borélienne $\mathcal{E}$. Étant donnée une fonction $U>0$ sur $E$, on notera $\mathcal{B}_{U}$ l'espace des fonctions $f$ mesurables sur $E$, à valeurs complexes, vérifiant

$$
\|f\|_{U}=\sup \left\{U(x)^{-1}|f(x)|, x \in E\right\}<+\infty .
$$

Soit $x_{0} \in E$ quelconque, et soit $V$ une fonction mesurable de $E$ dans $[1,+\infty$ [ telle que $V(x) \rightarrow+\infty$ quand $d\left(x, x_{0}\right) \rightarrow+\infty$. On suppose dans ce paragraphe que $\left(X_{n}\right)_{n \geqslant 0}$ est une chaîne $V$-géométriquement ergodique. Rappelons que cette propriété est par définition satisfaite s'il existe une probabilité $Q$-invariante, $v$, telle que $v(V)<+\infty$, si $\|Q V\|_{V}<+\infty$, et enfin si $Q$ vérifie la condition $(\mathrm{F} 2)$ sur $\mathcal{B}_{V}$. Des critères d'ergodicité $V$-géométrique sont présentés dans [18] (Chap. 16).

Soit $\lambda$ une mesure positive $\sigma$-finie sur $E$. En vue d'assurer la condition (F3) (Lemme 3.1), on suppose que $Q$ est de la forme $Q(x, d y)=K(x, y) d \lambda(y)$. Enfin on suppose que $\left(X_{n}\right)_{n \geqslant 0}$ est $\lambda$-irréductible et apériodique [18]. Ces deux conditions sont par exemple satisfaites si $K(x, y)>0$ pour tout $x \in E$ et pour $\lambda$-presque tout $y \in E$.

Proposition 3.1. Soit $W=\sqrt{V}$. Si $\xi \in \mathcal{B}_{W}$, alors $\left(\xi\left(X_{n}\right)\right)_{n} \geqslant 0$ vérifie le t.l.c de la Proposition 2.2. Si en outre $\sigma^{2}>0$ et $\mu_{0}(V)<+\infty$, alors avec $\mathcal{B}=\mathcal{B}_{W}$, on a (TLC), puis (TLL) si $\xi$ est non-arithmétique (le critère de non-arithméticité de la Remarque 2.6 s'applique ici).

Dans la preuve ci-dessous, nous montrons que $Q$ vérifie l'hypothèse $(\widetilde{\mathcal{F}})$ avec $\mathcal{B}=\mathcal{B}_{W}$ et $\widetilde{\mathcal{B}}=\mathcal{B}_{V}$. En particulier nous verrons que $Q(t) \rightarrow Q$ quand $t \rightarrow 0$ dans $\mathcal{L}\left(\mathcal{B}_{W}, \mathcal{B}_{V}\right)$; on observera que cette propriété n'est pas satisfaite a priori dans $\mathcal{L}\left(\mathcal{B}_{W}\right)$.

Preuve. Comme $v(V)<+\infty, \mathcal{B}=\mathcal{B}_{W}$ s'envoie continûment dans $\mathbb{L}^{2}(v)$ (cf. (F1) avec $q=2$ ). Par ailleurs $\left(X_{n}\right)_{n}$ est aussi $W$-géométriquement ergodique, c'est-à-dire $Q$ vérifie $(\mathrm{F} 2)$ sur $\mathcal{B}=\mathcal{B}_{W}$ [18] (Lemme 15.2.9 et Théorème 16.0.1). Par conséquent, si $\xi \in \mathcal{B}_{W}$, la Proposition 2.2 s'applique. En outre comme $\mathcal{B}_{W}$ vérifie la propriété $(*)$ (Rq. 2.5), on a (F4), et (F5) avec $I=\mathbb{R}$, sur $\mathcal{B}_{W}$.

Soit $\widetilde{\mathcal{B}}=\mathcal{B}_{V}$. Par hypothèse $Q$ vérifie (F2) sur cet espace. De plus, en utilisant l'inégalité $\left|\mathrm{e}^{i t \xi}-1\right| \leqslant|t||\xi|$, on a pour $f \in \mathcal{B}_{W}$ :

$$
|Q(t) f-Q f| \leqslant Q\left(\left|\mathrm{e}^{i t \xi}-1\right||f|\right) \leqslant|t| Q(|\xi| W)\|f\|_{W} \leqslant|t|\|\xi\|_{W} Q\left(W^{2}\right)\|f\|_{W},
$$

d'où $\|Q(t) f-Q f\|_{V} \leqslant|t|\|\xi\|_{W}\|Q\|_{V}\|f\|_{W}$. Il reste à établir que $Q$ vérifie (F3) sur $\mathcal{B}_{W}$.

Commençons par prouver que $Q$ est un opérateur compact de $\mathbb{L}^{\infty}(\lambda)$ dans $\mathbb{L}^{1}(v)$. Le noyau $K$ est clairement intégrable pour la mesure produit $\nu \otimes \lambda$, par conséquent $K$ peut être approché dans $\mathbb{L}^{1}(\nu \otimes \lambda)$ par des combinaisons linéaires finies de fonctions indicatrices $1_{A_{i} \times B_{j}}$, avec $A_{i}, B_{j} \in \mathcal{E}$. Il vient que $Q$ est limite, dans l'espace des opérateurs bornés de $\mathbb{L}^{\infty}(\lambda)$ dans $\mathbb{L}^{1}(v)$, d'opérateurs de rang fini. Ceci démontre le résultat annoncé.

Désignons maintenant par $\mathcal{B}_{0}$ l'espace des fonctions mesurables bornées sur $E$, muni de la norme de la convergence uniforme. Comme $\mathcal{B}_{0}$ s'envoie continûment dans $\mathbb{L}^{\infty}(\lambda)$, il vient que $Q$ est compact de $\mathcal{B}_{0}$ dans $\mathbb{L}^{1}(v)$. On peut maintenant établir (F3) :

Lemme 3.1. $Q\left(\left\{f \in \mathcal{B}_{W},\|f\|_{W} \leqslant 1\right\}\right)$ est relativement compacte dans $\left(\mathcal{B}_{W}, v(|\cdot|)\right)$.

Preuve. Soit $\left(f_{n}\right)_{n}$ une suite de fonctions de $\mathcal{B}_{W}$ telles que $\left\|f_{n}\right\|_{W} \leqslant 1$. On pose $g_{n}=Q f_{n}$ et, pour $k \geqslant 1, f_{n}^{(k)}=$ $f_{n} 1_{\left[\left|f_{n}\right| \leqslant k\right]}$ et $g_{n}^{(k)}=Q f_{n}^{(k)}$. Pour $k$ fixé, $\left(f_{n}^{(k)}\right)_{n}$ est une suite de fonctions qui sont toutes bornées sur $E$ par $k$. Par 
extraction successive de sous-suites, il existe donc des applications $\phi_{i}, i \geqslant 1$, strictement croissantes de $\mathbb{N}^{*}$ dans $\mathbb{N}^{*}$ telles que, pour tout $k \geqslant 1$, la suite $\left(g_{\phi_{1} \circ \cdots \circ \phi_{k}(n)}^{(k)}\right)_{n}$ converge dans $\mathbb{L}^{1}(v)$. Posons $\psi_{\ell}=\phi_{1} \circ \cdots \circ \phi_{\ell}$. Pour $q, p \geqslant k$, on a

$$
\left|g_{\psi_{q}(q)}-g_{\psi_{p}(p)}\right| \leqslant Q\left|f_{\psi_{q}(q)}-f_{\psi_{q}(q)}^{(k)}\right|+\left|g_{\psi_{q}(q)}^{(k)}-g_{\psi_{p}(p)}^{(k)}\right|+Q\left|f_{\psi_{p}(p)}^{(k)}-f_{\psi_{p}(p)}\right| .
$$

Or, pour tout $n \geqslant 1$, on a $v\left(Q\left|f_{n}-f_{n}^{(k)}\right|\right)=v\left(\left|f_{n}-f_{n}^{(k)}\right|\right)=v\left(\left|f_{n}\right| 1_{\left[\left|f_{n}\right|>k\right]}\right) \leqslant v\left(W 1_{[W>k]}\right)$ car $v$ est invariante et $\left|f_{n}\right| \leqslant W$.

Soit $\varepsilon>0$. Comme $v(W)<+\infty$, on a $v\left(W 1_{[W>k]}\right) \leqslant \frac{\varepsilon}{3}$ pour $k$ suffisament grand désormais fixé. Soit $N \geqslant 1$ tel que, pour $\ell, m \geqslant N$, on ait

$$
v\left(\left|g_{\psi_{k}(\ell)}^{(k)}-g_{\psi_{k}(m)}^{(k)}\right|\right) \leqslant \frac{\varepsilon}{3} .
$$

Pour $q \geqslant k$ et $p \geqslant k$, les entiers $\psi_{q}(q)$ et $\psi_{p}(p)$ sont de la forme $\psi_{k}\left(n_{q}\right)$ et $\psi_{k}\left(n_{p}\right)$ avec $n_{q} \geqslant q$ et $n_{p} \geqslant p$. On conclut de tout ce qui précède que, pour $q, p \geqslant \max \{k, N\}$, on a

$$
v\left(\left|g_{\psi_{q}(q)}-g_{\psi_{p}(p)}\right|\right) \leqslant \varepsilon .
$$

Il vient que la suite $\left(g_{\psi_{n}(n)}\right)_{n}$ converge dans $\mathbb{L}^{1}(v)$. Soit $g$ une fonction $v$-intégrable sur $E$ telle que lim $v_{n} v\left(\mid g_{\psi_{n}}(n)-\right.$ $g \mid)=0$. Comme $\left|g_{k}(x)\right| \leqslant\|Q\|_{W} W(x)$ pour tous $x \in E$ et $k \geqslant 1$, il existe une partie $A$ dans $\mathcal{E}$ telle que $v(A)=1$ et $|g(x)| \leqslant\|Q\|_{W} W(x)$ pour tout $x \in A$. Posons $h(x)=g(x)$ si $x \in A$, et $h(x)=0$ sinon. Alors $h \in \mathcal{B}_{W}$, et $\lim _{n} v\left(\left|g_{\psi_{n}(n)}-h\right|\right)=0$.

\subsubsection{Exemple d'une marche aléatoire}

Soit $\left(X_{n}\right)_{n \geqslant 0}$ la marche aléatoire sur $E=\mathbb{N}$ définie par la donnée d'une v.a $X_{0}$ sur $\mathbb{N}$, puis, pour $n \geqslant 1$, par $X_{n}=\max \left\{0, X_{n-1}+Y_{n}\right\}$, où $\left(Y_{n}\right)_{n \geqslant 1}$ est une suite de v.a.i.i.d. à valeurs dans $\mathbb{Z}$, indépendantes de $X_{0}$. On trouvera dans [18] des énoncés de t.l.c pour $\left(X_{n}\right)_{n}$ sous des hypothèses très générales. Nous nous intéressons ici uniquement au théorème local. Soit $\pi_{k}=\mathbb{P}\left(\left[Y_{1}=k\right]\right), k \in \mathbb{Z}$. On suppose qu'il existe $\gamma>0$ tel que

$$
\sum_{k \in \mathbb{Z}} k \pi_{k}<0 \quad \text { et } \sum_{k \geqslant 0} \pi_{k} \mathrm{e}^{\gamma k}<+\infty .
$$

Sous ces hypothèses, un théorème local a été établi dans [26] pour $\xi$ bornée. En fait cet énoncé est vérifié pour une classe de fonctions $\xi$ beaucoup plus générale. En effet, pour $s>0$ et $j \in \mathbb{N}$, posons $V_{s}(j)=\mathrm{e}^{s j}$. Alors il existe $0<\gamma_{0} \leqslant \gamma$ tel que $\left(X_{n}\right)_{n \geqslant 0}$ soit $V_{\gamma_{0}}$-géométriquement ergodique, voir [18] (p. 389). Afin d'assurer l'irréductibilité au sens de [18], supposons que $\pi_{k}>0$ pour tout $k \in \mathbb{Z}$ (on peut bien sûr affaiblir cette condition).

Sous ces hypothèses, la Proposition 3.1 s'applique avec $V=V_{\gamma_{0}}$.

\subsubsection{Exemple des modèles autorégressifs fonctionnels}

Soit $\left(X_{n}\right)_{n} \geqslant 0$ un processus autorégressif fonctionnel sur $\mathbb{R}^{d}$ défini par la donnée d'une v.a $X_{0}$ sur $\mathbb{R}^{d}$, puis par $X_{n}=\phi\left(X_{n-1}\right)+Y_{n}$ pour $n \geqslant 1$, où $\left(Y_{n}\right)_{n \geqslant 1}$ est une suite de v.a.i.i.d. à valeurs dans $\mathbb{R}^{d}$, indépendantes de $X_{0}$, et où $\phi$ est une fonction de l'espace $\mathbb{R}^{d}$ dans lui-même. Supposons que la loi commune des v.a $Y_{n}$ admette une densité $p>0$ et un moment d'ordre $\gamma_{0}>0$, et que $\phi$ soit continue et telle que $\lim \sup \left(\|x\|^{-1}\|\phi(x)\|\right)<1$ quand $\|x\| \rightarrow+\infty$.

Sous ces hypothèses, la Proposition 3.1 s'applique avec $V(x)=(1+\|x\|)^{\gamma_{0}}$.

En effet le noyau $Q$ est défini par $(Q f)(x)=\int_{\mathbb{R}^{d}} f(\phi(x)+y) p(y) d y$. Comme $p>0,\left(X_{n}\right)_{n} \geqslant 0$ est apériodique et Lebesgue-irréductible. En outre, grâce au théorème de convergence dominée, on a lim $\sup V(x)^{-1} Q V(x)<1$ quand $\|x\| \rightarrow+\infty$. Des résultats de [18] (Prop. 6.2.8 et Th. 16.0.1), on déduit que $\left(X_{n}\right)_{n \geqslant 0}$ est $V$-géométriquement ergodique. Enfin on a clairement $Q(x, d y)=K(x, y) d y$ avec $K(x, y)=p(y-\phi(x))$.

Dans le paragraphe suivant, en renforçant les conditions de régularité sur $\phi$ et $\xi$, on généralisera l'énoncé précédent au cas où $Y_{k}$ ne possède pas de densité. 


\subsection{Application aux Modèles itératifs Lipschitziens}

$(E, d)$ est à nouveau un espace métrique non compact dont toute boule fermée est compacte, et $x_{0}$ est un point quelconque de $E$. On désigne par $G$ un semi-groupe de transformations lipschitziennes de $E$, et par $\mathcal{G}$ une tribu sur $G$. On suppose que l'action de $G$ sur $E$ est mesurable.

Soit $\left(Y_{n}\right)_{n} \geqslant 1$ une suite de v.a.i.i.d. à valeurs dans $G$. On note $\pi$ leur loi commune. Étant donnée une variable aléatoire $X_{0}$ à valeurs dans $E$, de loi $\mu_{0}$, indépendante des v.a $Y_{n}$, on considère la suite $\left(X_{n}\right)_{n \geqslant 0}$ définie pour $n \geqslant 1$ par

$$
X_{n}=Y_{n} X_{n-1}, \quad \text { soit encore } X_{n}=Y_{n} \cdots Y_{1} X_{0} .
$$

Il est facile de voir que $\left(X_{n}\right)_{n \geqslant 0}$ est une chaîne de Markov de probabilité de transition définie par

$$
(Q f)(x)=\int_{G} f(g x) d \pi(g) .
$$

Ce cadre contient clairement celui des modèles itératifs Lipschitziens définis par exemple dans [6]. Par ailleurs, il comprend aussi le cas des itérations $\alpha$-Hölderiennes, $\alpha \in] 0,1]$, car $d(\cdot, \cdot)^{\alpha}$ est une distance sur $E$.

Nous donnons maintenant deux exemples d'applications du théorème $2.1^{\prime}$. Le premier, présenté dans le cadre général précédent, repose sur un t.l.c établi par M. Benda [2] dans le cas où $\xi$ est uniformément lipschitzienne. Le second, qui s'inscrit dans l'étude des produits de matrices aléatoires positives, est fondé sur un t.l.c démontré par H. Hennion [11].

Pour ces deux exemples, la propriété (TLL), lorsqu'elle est établie à l'aide du théorème standard de perturbation d'opérateurs, requiert des conditions de moments exponentiels pour la fonction $\delta(\cdot)$ définie ci-dessous, voir [12]. Dans l'article [13], qui utilise déjà les résultats de [16], ces conditions ont été remplacées par des hypothèses de moments polynomiaux ; celles-ci sont sensiblement améliorées dans les Propositions 3.2 et 3.3 ci-dessous.

Pour $g \in G$, on utilisera dans la suite les notations suivantes

$$
c(g)=\sup \left\{\frac{d(g x, g y)}{d(x, y)}, x, y \in E, x \neq y\right\} \quad \text { et } \quad \delta(g)=1+c(g)+d\left(g x_{0}, x_{0}\right) .
$$

Exemple 3.2.1 (Cas où $\xi$ est uniformément lipschitzienne). On suppose ici qu'il existe une constante $C \geqslant 0$ telle que l'on ait, pour $x, y \in E$,

$$
|\xi(x)-\xi(y)| \leqslant C d(x, y) .
$$

Il est établi dans [2] que, sous les hypothèses

$$
\int_{G} c(g)^{2} d \pi(g)<1 \text { et } \int_{G} d\left(g x_{0}, x_{0}\right)^{2} d \pi(g)<+\infty,
$$

la suite $\left(\frac{S_{n}}{\sqrt{n}}\right)_{n}$ converge en loi vers une gaussienne $\mathcal{N}\left(0, \sigma^{2}\right)$.

Nous renforçons ces hypothèses en supposant en outre que, pour un certain $\varepsilon>0$ et un entier $n_{0} \in \mathbb{N}^{*}$, on a

$$
\int_{G} \delta(g)^{2+\frac{\varepsilon}{2}} d \pi(g)<+\infty, \quad \int_{G} c(g) \delta(g)^{2+\varepsilon} d \pi(g)<+\infty, \quad \int_{G} c(g) \max \{c(g), 1\}^{2+\varepsilon} d \pi^{* n_{0}}(g)<1,
$$

où $\pi^{* n_{0}}$ est la loi de la v.a $Y_{n_{0}} \cdots Y_{1}$.

Les propriétés (TLC) et (TLL) dans la proposition ci-dessous peuvent être appliquées à $f \geqslant 0$ sur $E$ telle que $|f(x)-f(y)| \leqslant M d(x, y)\left(1+d\left(x, x_{0}\right)\right)\left(1+d\left(y, x_{0}\right)\right)$ pour tous $x, y \in E$, avec $M$ indépendant de $x, y$. 
Proposition 3.2. Sous ces hypothèses, il existe une unique probabilité $Q$-invariante, $v$, et $v\left(d\left(\cdot, x_{0}\right)^{2+\frac{\varepsilon}{2}}\right)<+\infty$. Si en outre $\sigma^{2}>0$ et $\mu_{0}\left(d\left(\cdot, x_{0}\right)^{2+\frac{\varepsilon}{2}}\right)<+\infty$, alors on a (TLC), puis (TLL) si $\xi$ est non-arithmétique.

Le critère de non-arithméticité de la Remarque 2.6 s'applique relativement à l'espace $\mathcal{B}=\mathcal{B}_{1}$ défini ci-dessous (cf. [13], Lemmes 9.1' et 9.1" ).

Exemple (Retour sur les modèles autorégressifs fonctionnels). Soit $\phi$ une contraction stricte sur $\mathbb{R}^{d}$, et soit $\left(X_{n}\right)_{n \geqslant 0}$ le processus autorégressif fonctionnel associé (voir Ex. 3.1.2). La loi commune $\pi$ des $Y_{k}$ est supposée ici quelconque.

$\left(X_{n}\right)_{n}$ est clairement défini selon le modèle ci-dessus, avec : $G=\mathbb{R}^{d}, g x=\phi(x)+g$, donc $c(g)$ est la constante de contraction de $\phi$, puis avec $x_{0}=0$, et $d(x, y)=\|x-y\|$ où $\|\cdot\|$ est une norme quelconque sur $\mathbb{R}^{d}$.

On dispose alors du résultat suivant : si $\xi$ est uniformément Lipschitzienne et si $\pi$ a un moment d'ordre $2+\varepsilon$, avec $\varepsilon>0$, alors la Proposition 3.2 s'applique.

Avant de présenter la preuve de la Proposition 3.2, nous définissons les espaces $\mathcal{B}_{\gamma}$ sur lesquels on fera opérer $Q$, et nous énonçons un lemme qui servira également dans l'Exemple 3.2.2.

Posons $p(x)=1+d\left(x, x_{0}\right)$, et, pour $\gamma>0$, désignons par $\mathcal{B}_{\gamma}$ l'espace des fonctions numériques $f$ sur $E$ telles que

$$
m_{\gamma}(f)=\sup \left\{\frac{|f(x)-f(y)|}{d(x, y) p(x)^{\gamma} p(y)^{\gamma}}, x, y \in E, x \neq y\right\}<+\infty .
$$

Pour $f \in \mathcal{B}_{\gamma}$, il est clair que $|f|_{\gamma}=\sup _{x \in E} \frac{|f(x)|}{p(x)^{\gamma+1}}<+\infty$. On pose

$$
\|f\|_{\gamma}=m_{\gamma}(f)+|f|_{\gamma} .
$$

On montre aisément que $\left(\mathcal{B}_{\gamma},\|\cdot\|_{\gamma}\right)$ est un espace de Banach. On notera que la condition imposée à $f$ dans les propriétés (TLC) et (TLL) de la Proposition 3.2 est $f \in \mathcal{B}_{1}$, et que, si $\mu$ est une probabilité sur $E$ telle que $\mu\left(d\left(\cdot, x_{0}\right)^{\gamma+1}\right)<+\infty$, alors $\mu \in \mathcal{B}_{\gamma}^{\prime}$.

Soit $\bar{\xi}$ une fonction réelle mesurable définie sur $G \times E$. On suppose qu'il existe une fonction $S$ mesurable sur $E$ telle que l'on ait, pour tous $x, y \in E$ et $g \in G$,

$$
\text { (S) }|\bar{\xi}(g, x)-\bar{\xi}(g, y)| \leqslant S(g) d(x, y) .
$$

Pour $t \in \mathbb{R}$, on pose

$$
P(t) f(x)=\int_{G} \mathrm{e}^{i t \bar{\xi}(g, x)} f(g x) d \pi(g)
$$

(on notera que $P(t)$ n'est pas a priori un noyau de Fourier associé à $Q$ ). Enfin, pour $\eta \geqslant 1$ et $n \in \mathbb{N}^{*}$, on définit

$$
\mathcal{M}_{\eta}=\int_{G} \delta(g)^{\eta} d \pi(g), \quad \mathcal{M}_{\eta}^{\prime}=\int_{G} c(g) \delta(g)^{\eta-1} d \pi(g), \quad \mathcal{C}_{\eta}^{(n)}=\int_{G} c(g) \max \{c(g), 1\}^{\eta-1} d \pi^{* n}(g) .
$$

On suppose pour le lemme suivant qu'il existe un réel $\gamma_{0}>1$ et un entier $n_{0} \in \mathbb{N}^{*}$ tels que l'on ait $\mathcal{M}_{\gamma_{0}+1}<+\infty$, $\mathcal{M}_{2 \gamma_{0}+1}^{\prime}<+\infty$, puis $\mathcal{C}_{2 \gamma_{0}+1}^{\left(n_{0}\right)}<1$, et enfin que $\int_{G} S(g) \delta(g)^{2} d \pi(g)<+\infty$.

Lemme 3.2. Sous ces hypothèses, il existe une unique probabilité $Q$-invariante, $v$, et $\int_{E} d\left(x, x_{0}\right)^{\gamma_{0}+1} d v(x)<+\infty$. En outre le triplet $(v, Q, P(\cdot))$ vérifie $\left(\widetilde{\mathcal{F}}^{\prime}\right)^{13}$ relativement aux espaces $\mathcal{B}=\mathcal{B}_{1}$ et $\widetilde{\mathcal{B}}=\mathcal{B}_{\gamma_{0}}$.

\footnotetext{
$\overline{13}$ Les hypothèses fonctionnelles du paragraphe 2 ont été définies relativement au noyaux de Fourier $Q(t)$, mais elles conservent un sens évident lorsque ceux-ci sont remplacés par des opérateurs du type $P(t)$.
} 
Le Lemme 3.2 est une conséquence directe de certains résultats établis dans [13]. ${ }^{14}$

Preuve de la Proposition 3.2. Soit $\bar{\xi}(g, x)=\xi(g x)$. Observons que les noyaux $P(t)$ coïncident ici avec les noyaux de Fourier $Q(t)$. Soit $\gamma_{0}=1+\frac{\varepsilon}{2}$. Les conditions de la Proposition 3.2 s'écrivent alors $\mathcal{M}_{\gamma_{0}+1}<+\infty$, $\mathcal{M}_{2 \gamma_{0}+1}^{\prime}<+\infty, \mathcal{C}_{2 \gamma_{0}+1}^{\left(n_{0}\right)}<1$. De plus, comme $\xi$ est uniformément Lipschitzienne, $\bar{\xi}$ vérifie $(\mathcal{S})$ avec $S(g)=C c(g)$. Il vient $\int_{G} S(g) \delta(g)^{2} d \pi(g) \leqslant C \int_{G} c(g) \delta(g)^{2 \gamma_{0}} d \pi(g)=\mathcal{M}_{2 \gamma_{0}+1}^{\prime}<+\infty$. Ainsi le lemme 3.2 s'applique. Comme la propriété $(\mathcal{P})$ est assurée grâce à [2], la Proposition 3.2 découle du Théorème 2.1' .

Exemple 3.2.2 (Produits de matrices aléatoires positives). $G$ est ici le semi-groupe des matrices d'ordre $d$, à coefficients $\geqslant 0$, dont chaque ligne et chaque colonne contiennent un coefficient $>0$, et $E$ est l'ensemble défini par

$$
E=\left\{x \in\left(\mathbb{R}_{+}^{*}\right)^{d},\|x\|=1\right\},
$$

où l'on a posé $\|x\|=\sum_{k=1}^{d}\left|x_{k}\right|$. Pour $g \in G$ et $x \in \mathbb{R}^{d}$, on note $g(x)$ l'image de $x$ par $g$, puis $g^{*}$ la matrice transposée de $g$, et enfin

$$
\|g\|=\sup \{\|g(y)\|: y \in E\} \quad \text { et } \quad v(g)=\inf \{\|g(y)\|: y \in E\} .
$$

Soit $\left(Y_{k}\right)_{k \geqslant 1}$ une suite de v.a.i.i.d. à valeurs dans $G$, de loi commune notée $\pi$, et enfin soit $R_{n}=Y_{n} \cdots Y_{1}$, avec la convention $R_{0}=I d_{E}$. H. Hennion a établi dans [11] que, si

$$
\int_{G}\left(\left|\ln \left\|g^{*}\right\|\right|+\left|\ln v\left(g^{*}\right)\right|\right)^{2} d \pi(g)<+\infty,
$$

et si, pour un certain $n_{0} \in \mathbb{N}^{*}$, le support de la loi de la v.a $R_{n_{0}}$ contient une matrice à coefficients $>0$, alors il existe $m_{1} \in \mathbb{R}$ et $\sigma^{2} \in \mathbb{R}_{+}$tels que, pour tout $y \in E$, la suite $\left(\frac{1}{\sqrt{n}}\left(\ln \left\|R_{n}(y)\right\|-n m_{1}\right)\right)_{n} \geqslant 1$ converge en loi vers une gaussienne $\mathcal{N}\left(0, \sigma^{2}\right)$.

Soit $X_{0}$ une v.a. à valeurs dans $E$, de loi $\mu_{0}$ quelconque, indépendante des v.a $Y_{k}$. Il est facile de voir que le t.l.c précédent s'applique encore à $R_{n}\left(X_{0}\right)$. Comme dans [11] et [13], nous considérons sur $E$ la distance de Hilbert, notée $d_{H}$.

Enfin nous renforçons les hypothèses précédentes en supposant que, pour un certain $\varepsilon>0$,

$$
\int_{G}\left(\left|\ln \left\|g^{*}\right\|\right|+\left|\ln v\left(g^{*}\right)\right|\right)^{2+\varepsilon} d \pi(g)<+\infty,
$$

et que, pour un certain $n_{0} \in \mathbb{N}^{*}$, le support de la loi de $R_{n_{0}}$ contient deux matrices $g_{1}, g_{2}$ à coefficients $>0$, dont les rayons spectraux respectifs $\rho_{1}, \rho_{2}$ vérifient $\ln \frac{\rho_{2}}{\rho_{1}} \notin \mathbb{Q}$.

L'action de $G$ sur $E$ est définie par $g x=g(x) /\|g(x)\|$.

\footnotetext{
$\overline{14}$ Plus précisément
}

(i) L'existence de $v$, son unicité et la condition de moment, sont établis dans [13, Th. I].

Il vient que $\mathcal{B}_{1}$ s'envoie continûment dans $\mathbb{L}^{q}(v)$, avec $q=\frac{\gamma_{0}+1}{2}$, et $v \in \mathcal{B}_{\gamma}^{\prime}$ pour tout $0<\gamma \leqslant \gamma_{0}$.

(ii) $Q$ vérifie (F2) sur $\mathcal{B}_{\gamma}$ pour tout $0<\gamma \leqslant \gamma_{0}[13$, Th. 5.5].

(iii) L'injection canonique de $\left(\mathcal{B}_{1},\|\cdot\|_{1}\right)$ dans $\left(\mathcal{B}_{1}, v(|\cdot|)\right)$ est compacte [13, Lemme 5.4] et pour $t \in \mathbb{R}, P(t)$ opère continûment sur $\mathcal{B}_{1}$ [13, Lemme 6.1]. Donc $P(t)$ est compact de $\left(\mathcal{B}_{1},\|\cdot\|_{1}\right)$ dans $\left(\mathcal{B}_{1}, v(|\cdot|)\right)$.

(iv) $P(\cdot)$ vérifie la condition (F5) sur $\mathcal{B}_{1}[13$, Lemme 6.1].

(v) Si $\gamma_{0}>1$, alors quand $t \rightarrow 0, Q(t)$ converge vers $Q$ dans $\mathcal{L}\left(\mathcal{B}_{1}, \mathcal{B}_{\gamma_{0}}\right)$ [13, Lemme 6.2]. 
Proposition 3.3. Sous ces hypothèses il existe sur $E$ une unique probabilité $Q$-invariante, $v$, et l'on a $v\left(d_{H}\left(\cdot, x_{0}\right)^{2+\frac{\varepsilon}{2}}\right)<+\infty$. Si en outre le réel $\sigma^{2}$ ci-dessus est $>0$ et si l'on a $\mu_{0}\left(d_{H}\left(\cdot, x_{0}\right)^{2+\frac{\varepsilon}{2}}\right)<+\infty$, alors on a pour toute fonction réelle $\psi$ continue bornée sur $\mathbb{R}$, à support compact

$$
\lim _{n} \sigma \sqrt{2 \pi n} \mathbb{E}\left[\psi\left(\ln \left\|R_{n}\left(X_{0}\right)\right\|-n m_{1}\right)\right]=L(\psi) .
$$

Preuve. Vis-à-vis de l'action de $G$ sur $E$ définie ci-dessus, la fonction $a(g, x)=\ln \|g(x)\|$ est un cocycle additif :

$$
a\left(g g^{\prime}, x\right)=a\left(g, g^{\prime} x\right)+a\left(g^{\prime}, x\right) .
$$

Par conséquent, en posant $\bar{\xi}(g, x)=a(g, x)-m_{1}$, il vient que

$$
\ln \left\|R_{n}\left(X_{0}\right)\right\|-n m_{1}=\sum_{k=1}^{n} \bar{\xi}\left(Y_{k}, R_{k-1} X_{0}\right)=\sum_{k=1}^{n} \bar{\xi}\left(\bar{X}_{k}\right),
$$

où $\left(\bar{X}_{n}\right)_{n \geqslant 0}$ est la chaîne de Markov définie sur l'espace $\bar{E}=G \times E$ par $\bar{X}_{0}=\left(I d_{E}, X_{0}\right)$, puis par $\bar{X}_{n}=$ $\left(Y_{n}, R_{n-1} X_{0}\right)$; sa probabilité de transition est $\bar{Q} F(g, x)=\int_{G} F(h, g x) d \pi(h)$.

La conclusion de la Proposition 3.3 correspond donc à la propriété (TLL) pour la chaîne $\left(\bar{X}_{n}\right)_{n \geqslant 0}$ et la fonction $\bar{\xi}$.

Nous allons démontrer qu'il existe sur $\bar{E}$ une unique probabilité $\bar{Q}$-invariante, $\bar{v}$, puis que $\bar{Q}$ et les noyaux de Fourier $\bar{Q}(t)$ associés à $\bar{Q}$ et $\bar{\xi}$ vérifient $\left(\widetilde{\mathcal{F}}^{\prime}\right)$ vis-à-vis d'espaces $\overline{\mathcal{B}}_{1}$ et $\overline{\mathcal{B}}_{1+\frac{\varepsilon}{2}}$ construits plus bas. À cet effet nous conservons les notations du Lemme 3.2 en associant à $\pi$ et $\bar{\xi}$ les noyaux $Q f(x)=\int_{G} f(g x) d \pi(g)$ et $P(t) f(x)=$ $\int_{G} \mathrm{e}^{i t \bar{\xi}(g, x)} f(g x) d \pi(g)$, puis en considérant les espaces $\mathcal{B}_{\gamma}$ définis ici avec $d=d_{H}$.

Lemme 3.3. Les hypothèses de la Proposition 3.3 impliquent celles du Lemme 3.2 avec $\gamma_{0}=1+\frac{\varepsilon}{2}$.

Admettons pour le moment ce lemme, et indiquons comment les propriétés du Lemme 3.2, vérifiées pour $(Q, P(\cdot))$ s'étendent à $(\bar{Q}, \bar{Q}(\cdot))$. Tout d'abord pour passer de l'espace d'états $E$ à $\bar{E}$, nous posons $J f(g, x)=$ $f(g x)$ pour $f$ borélienne sur $E, x \in E, g \in G$, et nous définissons l'espace $\overline{\mathcal{B}}_{\gamma}=J\left(\mathcal{B}_{\gamma}\right)$, muni de la norme $\|J f\|_{\gamma}=\|f\|_{\gamma}$. Il est facile de voir que $\overline{\mathcal{B}}_{\gamma}$ est un espace de Banach, et que $\bar{Q}$ et $\bar{Q}(t)$ vérifient, pour tous $n \geqslant 1$ et $t \in \mathbb{R}$

$$
(\bar{Q})^{n} \circ J=J \circ Q^{n} \quad \text { et } \quad \bar{Q}(t)^{n} \circ J=J \circ P(t)^{n} .
$$

On voit aisément que la probabilité $\bar{v}=\pi \times v$ est $\bar{Q}$-invariante [12, Cor. X.8]. Comme $v$ a un moment d'ordre $2+\frac{\varepsilon}{2}$ (Lemme 3.2), on a $v \in\left(\mathcal{B}_{1+\frac{\varepsilon}{2}}\right)^{\prime}$, et il vient que $\bar{v}$ définit une forme linéaire sur $\overline{\mathcal{B}}_{1+\frac{\varepsilon}{2}}$. De la même façon, on peut montrer, grâce à la condition de moment sur $\mu_{0}$, que la loi de $\bar{X}_{0}=\left(I d_{E}, X_{0}\right)$ définit une forme linéaire continue $\operatorname{sur} \overline{\mathcal{B}}_{1+\frac{\varepsilon}{2}}$. En outre, grâce aux formules ci-dessus, il est très facile de voir que $\left(\widetilde{\mathcal{F}}^{\prime}\right)$ se transpose à $(\bar{v}, \bar{Q}, \bar{Q}(t))$ vis-à-vis des espaces $\overline{\mathcal{B}}_{1}$ et $\overline{\mathcal{B}}_{1+\frac{\varepsilon}{2}}$.

Enfin l'hypothèse sur les matrices $g_{1}, g_{2}$ assure que $\bar{\xi}$ vérifie le critère de non-arithméticité de la Proposition 2.4 (voir [13, § 3.3]), et en vertu de $(* *)$ et des résultats de [11], la suite $\left(\bar{\xi}\left(\bar{X}_{k}\right)\right)_{k}$ vérifie l'hypothèse $(\mathcal{P})$ lorsque le réel $\sigma^{2}$ de [11] est $>0$. La Proposition 3.3 découle alors du Théorème $2.1^{\prime}$.

Preuve du Lemme 3.3. Les espaces $\mathcal{B}_{\gamma}$ et les nombres $c(g), \delta(g)$ sont ici définis avec la distance de Hilbert $d=d_{H}$. Grâce aux propriétés de $d_{H}$, on sait que $c(\cdot) \leqslant 1$, puis que $c(g)<1$ si les coefficients de $g$ sont $>0$, voir $[1,11]$. Par hypothèse, une telle matrice existe dans le support de la loi de $R_{n_{0}}$, et ceci assure que $\mathcal{C}_{\eta}^{\left(n_{0}\right)}<1$ pour tout $\eta \geqslant 1$.

La condition intégrale de la Proposition 3.3 entraîne $\mathcal{M}_{2+\varepsilon}<+\infty$ (voir [13, § 3.3]). Avec $\gamma_{0}=1+\frac{\varepsilon}{2}$ il vient $\mathcal{M}_{\gamma_{0}+1}=\mathcal{M}_{2+\frac{\varepsilon}{2}}<+\infty$, puis $\mathcal{M}_{2 \gamma_{0}+1}^{\prime} \leqslant \mathcal{M}_{2+\varepsilon}<+\infty$ car $c(\cdot) \leqslant 1$. En outre on montre dans [13, § 3.3] que 
la fonction $\bar{\xi}$ ci-dessus vérifie $(\mathcal{S})$ avec $S=1$, d'où $\int_{G} S(g) \delta(g)^{2} d \pi(g)=\int_{G} \delta(g)^{2} d \pi(g) \leqslant \mathcal{M}_{2+\varepsilon}<+\infty$. Les conditions du Lemme 3.2 sont donc satisfaites.

\section{Preuve des énoncés $2.1,2.1^{\prime}$, et 2.4}

\subsection{Théorème de Keller-Liverani}

Pour simplifier nous présentons les résultats de [16] dans le cadre de ce papier, à savoir : la famille d'opérateurs est donnée par les noyaux de Fourier $Q(t)$, et la semi-norme auxiliaire sur $\mathcal{B}$ est $v(|\cdot|) .{ }^{15}$

Théorème (K-L) [16]. Supposons que Q vérifie (F2), et que les hypothèses (i)-(iv) suivantes soient satisfaites:

(i) Pour $t \in \mathbb{R}, Q(t) \in \mathcal{L}(\mathcal{B})$, et pour $n \geqslant 1$ et $f \in \mathcal{B}$, on a $v\left(\left|Q(t)^{n} f\right|\right) \leqslant v(|f|)$.

(ii) $Q(\cdot)$ vérifie la condition $(\mathrm{F} 5)$ sur un certain intervalle ouvert I contenant $t=0$.

(iii) Pour $t \in I$, le rayon spectral essentiel de $Q(t)$ est $\leqslant$ au réel $\kappa$ intervenant dans (F5).

(iv) Il existe sur I une fonction réelle continue $\varepsilon(\cdot)$, nulle en $t=0$, telle que

$$
v(|Q(t) f-Q f|) \leqslant \varepsilon(t)\|f\|
$$

Alors il existe un intervalle ouvert $J \subset I$ centré en $t=0$, et des applications $\lambda(\cdot), v(\cdot), \phi(\cdot)$ et $N(\cdot)$ à valeurs respectivement dans $\mathbb{C}, \mathcal{B}, \mathcal{B}^{\prime}$ et $\mathcal{L}(\mathcal{B})$ tels que l'on ait, pour $t \in J, n \geqslant 1$, et $f \in \mathcal{B}$,

$$
Q(t)^{n} f=\lambda(t)^{n}\langle\phi(t), f\rangle v(t)+N(t)^{n} f
$$

avec en outre les propriétés suivantes:

(a) $\langle\phi(t), v(t)\rangle=1, \phi(t) N(t)=0, N(t) v(t)=0$, et $\left\|N(t)^{n}\right\| \leqslant C \kappa^{n}$, où $C$ est une constante indépendante de $t \in J$ et $n \in \mathbb{N}^{*}$.

(b) $\lim _{t \rightarrow 0} \lambda(t)=1,\langle v, v(t)\rangle=1, \lim _{t \rightarrow 0}\langle v,|v(t)-\mathbf{1}|\rangle=0$, et il existe une fonction réelle continue $\vartheta(\cdot)$, nulle en $t=0$, telle que l'on ait pour $f \in \mathcal{B},|\langle\phi(t), f\rangle-v(f)| \leqslant \vartheta(t)\|f\|$.

La décomposition spectrale du théorème (K-L), puis l'assertion (a) et la 1ère propriété de (b), sont présentées dans les énoncés de [16]. Les autres points de (b) sont des conséquences assez directes de résultats intermédiaires contenus dans [16] dont nous rappelons les principaux arguments.

D'après [16], il existe une fonction réelle continue $\vartheta(\cdot)$, nulle en $t=0$, telle que l'on ait, pour tout $f \in \mathcal{B},|\langle\nu,|\Pi(t) f-\Pi(0) f|\rangle| \leqslant \vartheta(t)\|f\|$, où $\Pi(t)$ est un projecteur de rang 1 sur $\operatorname{ker}(Q(t)-\lambda(t))$ tel que $\Pi(0) f=v(f)$ 1. De plus l'élément propre dual $\phi(t)$ peut être défini par $\phi(t)=\Pi(t)^{*} v$, tandis que $v(t)=$ $\langle v, \Pi(t) \mathbf{1}\rangle^{-1} \Pi(t) \mathbf{1}$. La 2ième assertion de (b) en résulte. La 3ième est une conséquence de $\lim \langle v,| \Pi(t) \mathbf{1}-$ $\Pi(0) \mathbf{1}|\rangle=0$ et $\Pi(0) \mathbf{1}=\mathbf{1}$. Enfin on déduit la 4ième de l'égalité $\langle\phi(t), f\rangle=\langle v, \Pi(t) f\rangle$.

Le lemme suivant montre que le théorème précédent s'applique sous les conditions du $\S 2$.

Lemme 4.1. Sous les hypothèses (F1) (F3) (F4) (F5) [ou bien (F1) ( $\left.\mathrm{F}_{3,4}\right)$ (F5), cf. Rq. 2.4], les conditions (i)-(iv) du théorème (K-L) sont satisfaites.

En conséquence, sous les hypothèses $(\mathcal{F})$ ou $\left(\mathcal{F}^{\prime}\right)$, les conclusions du théorème $(\mathrm{K}-\mathrm{L})$ sont vérifiées; en outre la fonction $\lambda(\cdot)$ est continue sur $J$.

\footnotetext{
$\overline{15}$ Les résultats de [16] sont présentés avec une norme auxiliaire $|\cdot|$ sur $\mathcal{B}$ vérifiant $|\cdot| \leqslant\|\cdot\|$. Il est facile de voir que ceux-ci subsistent lorsque $|\cdot|$ est remplacée par une semi-norme.
} 
Preuve. Soit $t \in \mathbb{R}, n \geqslant 1$. On a $\left|Q(t)^{n} f\right| \leqslant Q^{n}|f|$, donc $v\left(\left|Q(t)^{n} f\right|\right) \leqslant v\left(Q^{n}|f|\right)=v(|f|)$, d'où le second point de (i) (cf. Rq. 2.4 pour le 1er point de (i)). La propriété (ii) correspond à (F5). Pour prouver (iii), observons que, grâce à $(\mathrm{F} 3)(\mathrm{F} 4)$ [ou bien $\left.\left(\mathrm{F}_{3,4}\right)\right], Q(t)$ est compact de $(\mathcal{B},\|\cdot\|)$ dans $(\mathcal{B}, v(|\cdot|))($ Rq. 2.4). De $(\mathrm{F} 5)$, on déduit alors du théorème de Hennion [10], fondé sur la formule de Nussbaum [22], que le rayon spectral essentiel de $Q(t)$ est $\leqslant \kappa$.

Pour prouver (iv), notons $p$ le nombre conjugué du réel $q$ de la condition (F1). En utilisant l'invariance de $v$, l'inégalité de Hölder et la propriété (F1), on a, pour $f \in \mathcal{B}$ et $t_{0}, h \in \mathbb{R}$

$$
v\left(\left|Q\left(t_{0}+h\right) f-Q\left(t_{0}\right) f\right|\right) \leqslant v\left(Q\left(\left|\mathrm{e}^{i h \xi}-1\right||f|\right)\right)=v\left(\left|\mathrm{e}^{i h \xi}-1\right||f|\right) \leqslant A \varepsilon(h)\|f\|
$$

avec $\varepsilon(h)=\left(v\left(\left|\mathrm{e}^{i h \xi}-1\right|^{p}\right)\right)^{1 / p}$ qui tend vers 0 avec $h$ en vertu du théorème de Lebesgue.

Pour établir la continuité de $\lambda(\cdot)$ sur l'intervalle $J$, il suffit d'observer que, pour $t_{0} \in J$ quelconque, la famille $\{Q(t), t \in \mathbb{R}\}$ vérifie les conditions (i)-(iii) du théorème de Keller-Liverani relativement à l'intervalle $J$. Ceci, adjoint à la propriété $\left(1^{\prime}\right)$, permet d'appliquer à nouveau le théorème de Keller-Liverani, mais cette fois-ci au voisinage de $t=t_{0}$; il vient que $\lambda(\cdot)$ est continue en $t_{0}$.

\subsection{Preuve de la Proposition 2.4}

La preuve de la Proposition 2.4 découle des points 1-3 ci-dessous. On désignera par $K$ une partie compacte quelconque de $\mathbb{R}^{*}$.

1. Pour tout $t \in \mathbb{R}^{*}$, on a $r(Q(t))<1$.

En itérant l'inégalité de Doeblin-Fortet (F5) et en utilisant le fait que $v\left(\left|Q(t)^{n} f\right|\right) \leqslant v(|f|)$, il vient que les itérés de $Q(t)^{N}$ sont uniformément bornés sur $(\mathcal{B},\|\cdot\|)$. Donc $r(Q(t)) \leqslant 1$. Par ailleurs, si l'on avait $r(Q(t))=1$, alors $Q(t)$ serait quasi-compact (cf. preuve de (iii) du th. (K-L)), et il existerait donc une fonction propre $w \in \mathcal{B}$ associée à $\lambda \in \mathbb{C}$ de module 1, ce qui est impossible sous la condition de la Proposition 2.4 (voir [12], Prop. V.2).

2. On a $r_{K}=\sup \{r(Q(t)), t \in K\}<1$.

Supposons que $r_{K}=1$. Alors il existe une suite $\left(\tau_{k}\right)_{k}$ dans $K$ telle que $\lim _{k} r\left(Q\left(\tau_{k}\right)\right)=1$. Pour $k \geqslant 1$, désignons par $\lambda_{k}$ une valeur spectrale de $Q\left(\tau_{k}\right)$ telle que $\left|\lambda_{k}\right|=r\left(Q\left(\tau_{k}\right)\right)$. Par compacité, on peut supposer que les suites $\left(\tau_{k}\right)_{k}$ et $\left(\lambda_{k}\right)_{k}$ convergent. Soient $t_{0}=\lim _{k} \tau_{k}$ et $\lambda=\lim _{k} \lambda_{k}$; observons que $t_{0} \in K$, donc $t_{0} \neq 0$, et $|\lambda|=1$. Par ailleurs, puisque par hypothèse on a $I=\mathbb{R}$ dans $(\mathrm{F} 5)$ [et dans $\left(\mathrm{F}_{3,4}\right)$ sous la condition $\left(\mathcal{F}^{\prime}\right)$ ], la famille $\{Q(t), t \in \mathbb{R}\}$, quand $t \rightarrow t_{0}$, vérifie les conditions du théorème de Keller-Liverani. On déduit alors de [16, p. 145] que $\lambda$ est une valeur spectrale de $Q\left(t_{0}\right)$, ce qui est absurde d'après le point $l$ car $t_{0} \neq 0$ et $|\lambda|=1$.

3. Il existe $c \geqslant 0$ et $\rho_{K}<1$ tels que l'on ait, pour tout $n \geqslant 1$, $\sup _{t \in K}\left\|Q(t)^{n}\right\| \leqslant c \rho_{K}^{n}$.

Soit $\rho_{K}$ tel que $r_{K}<\rho_{K}<1$, et soit $\Gamma$ le cercle orientée $\left\{|z|=\rho_{K}\right\}$ dans $\mathbb{C}$. On a

$$
Q(t)^{n}=\frac{1}{2 i \pi} \int_{\Gamma} z^{n}(z-Q(t))^{-1} d z .
$$

Les résultats de [16], appliqués comme en 2 au voisinage de $t_{0} \in K$, assurent qu'il existe un intervalle ouvert $I_{t_{0}}$ contenant $t_{0}$ tel que $\sup \left\{\left\|(z-Q(t))^{-1}\right\|, t \in I_{t_{0}},|z|=\rho_{K}\right\}<+\infty$. Par compacité il vient que sup $\{\|(z-$ $\left.Q(t))^{-1} \|, t \in K,|z|=\rho_{K}\right\}<+\infty$. La propriété 3 en résulte.

\subsection{Preuves des Théorèmes 2.1 et $2.1^{\prime}$}

Le lien entre la fonction caractéristique de $S_{n}$ et les éléments du théorème (K-L) découle des formules suivantes : pour tout $f \in \mathcal{B}$, toute probabilité $\mu \in \mathcal{B}^{\prime}$, et pour $n \geqslant 1$,

$$
\mathbb{E}_{\mu}\left[f\left(X_{n}\right) \mathrm{e}^{i t S_{n}}\right]=\left\langle\mu, Q(t)^{n} f\right\rangle=\lambda(t)^{n}\langle\phi(t), f\rangle\langle\mu, v(t)\rangle+\left\langle\mu, N(t)^{n} f\right\rangle .
$$


La première égalité, vraie pour tout $t \in \mathbb{R}$, se déduit aisément de la propriété de Markov, voir par exemple [12]; la seconde, vérifiée pour $t \in J$, est une conséquence du théorème (K-L).

Comme indiqué en introduction, l'hypothèse $(\mathcal{P})$ va permettre de préciser le comportement en $t=0$ de la valeur propre perturbée $\lambda(t)$.

Lemme 4.2. Sous les hypothèses du théorème $(\mathrm{K}-\mathrm{L})$ et sous la condition $(\mathcal{P})$, on a :

( $\alpha$ ) Pour tout $t \in \mathbb{R}, \lim _{n \rightarrow+\infty} \lambda\left(\frac{t}{\sigma \sqrt{n}}\right)^{n}=\mathrm{e}^{-t^{2} / 2}$,

( $\beta) \lambda(u)=1-\frac{\sigma^{2}}{2} u^{2}+\mathrm{o}\left(u^{2}\right)$.

Preuve. Grâce à un résultat classique, on sait d'après $(\mathcal{P})$ que la suite de fonctions caractéristiques $\left.\left(\mathbb{E}_{\nu}\left[\mathrm{e}^{i \frac{\dot{\sigma} \sqrt{n}}{\sigma} S_{n}}\right)_{n}\right]\right)_{n}$ converge uniformément sur tout compact de $\mathbb{R}$ vers $\phi$ donnée par $\phi(t)=\mathrm{e}^{-t^{2} / 2}$. Soit $N \in \mathbb{N}^{*}$ tel que $\frac{1}{\sigma \sqrt{N}} \in J$. Dans la suite on supposera toujours que $|t| \leqslant 1$ et $n \geqslant N$, de sorte que $\frac{t}{\sigma \sqrt{n}} \in J$. La formule (2) appliquée avec $f=v\left(\frac{t}{\sigma \sqrt{n}}\right)$ et $\mu=v$ montre que

$$
\lambda\left(\frac{t}{\sigma \sqrt{n}}\right)^{n}=\mathbb{E}_{\nu}\left[v\left(\frac{t}{\sigma \sqrt{n}}\right)\left(X_{n}\right) \mathrm{e}^{i \frac{t}{\sigma \sqrt{n}} S_{n}}\right],
$$

d'où en utilisant l'inégalité triangulaire et l'invariance de $v$,

$$
\begin{aligned}
\left|\lambda\left(\frac{t}{\sigma \sqrt{n}}\right)^{n}-\mathrm{e}^{-\frac{t^{2}}{2}}\right| & \leqslant \mathbb{E}_{\nu}\left[\left|v\left(\frac{t}{\sigma \sqrt{n}}\right)\left(X_{n}\right)-1\right|\right]+\left|\mathbb{E}_{\nu}\left[\mathrm{e}^{i \frac{t}{\sigma \sqrt{n}} S_{n}}\right]-\mathrm{e}^{-\frac{t^{2}}{2}}\right| \\
& =\left\langle v,\left|v\left(\frac{t}{\sigma \sqrt{n}}\right)-\mathbf{1}\right|\right\rangle+\left|\mathbb{E}_{\nu}\left[\mathrm{e}^{i \frac{t}{\sigma \sqrt{n}} S_{n}}\right]-\mathrm{e}^{-\frac{t^{2}}{2}}\right| .
\end{aligned}
$$

Du 3ième point de l'assertion (b) du théorème (K-L) et de la propriété rappelée plus haut, on déduit $(\alpha)$. Plus précisément soit $K=[-1,1]$. Il vient :

$$
\forall \eta>0, \exists N_{0}(\eta) \geqslant N, \forall n \geqslant N_{0}(\eta), \forall t \in K, \quad\left|\lambda\left(\frac{t}{\sigma \sqrt{n}}\right)^{n}-\mathrm{e}^{-\frac{t^{2}}{2}}\right| \leqslant \eta .
$$

Soient $t \in K$ et $n \geqslant N_{0}(\eta)$. On a $\mathrm{e}^{-1 / 2} \leqslant \mathrm{e}^{-t^{2} / 2} \leqslant 1$, d'où $\left|\lambda\left(\frac{t}{\sigma \sqrt{n}}\right)^{n}-1\right| \leqslant \eta+1-\mathrm{e}^{-1 / 2}$. Par conséquent, si $\eta \leqslant \frac{1}{2} \mathrm{e}^{-1 / 2}$, alors $\lambda\left(\frac{t}{\sigma \sqrt{n}}\right)^{n}$ et $\mathrm{e}^{-t^{2} / 2}$ sont dans le disque complexe $D$ de centre $z=1$ et de rayon $1-\frac{1}{2} \mathrm{e}^{-1 / 2}$. En outre, d'après le Lemme 4.1 , on peut écrire $\lambda(u)=|\lambda(u)| \mathrm{e}^{i \theta(u)}$, avec $\theta(\cdot)$ continue sur $J$ telle que $\theta(0)=0$. On a $\lambda\left(\frac{t}{\sigma \sqrt{n}}\right)^{n}=\left|\lambda\left(\frac{t}{\sigma \sqrt{n}}\right)\right|^{n} \mathrm{e}^{i n \theta\left(\frac{t}{\sigma \sqrt{n}}\right)}$, et par continuité on déduit de ce qui précède que $\left|n \theta\left(\frac{t}{\sigma \sqrt{n}}\right)\right|<\frac{\pi}{2}$. Pour $z \in \mathbb{C}$ non nul, posons $\log z=\ln |z|+i \arg (z)$, avec $\arg (z) \in]-\pi, \pi]$. Alors $\log \lambda\left(\frac{t}{\sigma \sqrt{n}}\right)^{n}=n \log \lambda\left(\frac{t}{\sigma \sqrt{n}}\right)$.

Soit $\varepsilon>0$. Comme la fonction log est lipschitzienne sur $D$, on obtient en choisissant $\eta=\eta(\varepsilon)$ convenablement :

$$
\exists N_{1}(\varepsilon) \geqslant N, \forall n \geqslant N_{1}(\varepsilon), \forall t \in K, \quad\left|n \log \lambda\left(\frac{t}{\sigma \sqrt{n}}\right)+\frac{t^{2}}{2}\right| \leqslant \frac{\varepsilon}{2} .
$$

Écrivons $z-1=(\log z)(1+\alpha(z))$ avec $\lim _{z \rightarrow 1} \alpha(z)=0$. Alors, pour $\varepsilon \leqslant 1, n \geqslant N_{1}(\varepsilon)$ et $t \in K$,

$$
\begin{aligned}
\left|n\left(\lambda\left(\frac{t}{\sigma \sqrt{n}}\right)-1\right)+\frac{t^{2}}{2}\right| & \leqslant n\left|\lambda\left(\frac{t}{\sigma \sqrt{n}}\right)-1-\log \lambda\left(\frac{t}{\sigma \sqrt{n}}\right)\right|+\left|n \log \lambda\left(\frac{t}{\sigma \sqrt{n}}\right)+\frac{t^{2}}{2}\right| \\
& \leqslant n\left|\log \lambda\left(\frac{t}{\sigma \sqrt{n}}\right)\right|\left|\alpha\left(\lambda\left(\frac{t}{\sigma \sqrt{n}}\right)\right)\right|+\frac{\varepsilon}{2} \\
& \leqslant\left(\frac{t^{2}}{2}+\frac{\varepsilon}{2}\right)\left|\alpha\left(\lambda\left(\frac{t}{\sigma \sqrt{n}}\right)\right)\right|+\frac{\varepsilon}{2}
\end{aligned}
$$




$$
\leqslant\left|\alpha\left(\lambda\left(\frac{t}{\sigma \sqrt{n}}\right)\right)\right|+\frac{\varepsilon}{2}
$$

Puisque $\lim _{u \rightarrow 0} \lambda(u)=1,\left(\lambda\left(\frac{\cdot}{\sigma \sqrt{n}}\right)\right)_{n}$ converge uniformément vers 1 sur $K$. Par conséquent :

$$
\exists N_{2}(\varepsilon) \geqslant N, \forall n \geqslant N_{2}(\varepsilon), \forall t \in K, \quad\left|\alpha\left(\lambda\left(\frac{t}{\sigma \sqrt{n}}\right)\right)\right| \leqslant \frac{\varepsilon}{2} .
$$

Finalement, en posant $N(\varepsilon)=\max \left\{N_{1}(\varepsilon), N_{2}(\varepsilon)\right\}$, on a montré que :

$$
\forall n \geqslant N(\varepsilon), \forall t \in K, \quad\left|n\left(\lambda\left(\frac{t}{\sigma \sqrt{n}}\right)-1\right)+\frac{t^{2}}{2}\right| \leqslant \varepsilon .
$$

Pour $\frac{1}{2} \leqslant|t| \leqslant 1$ et $n \geqslant N(\varepsilon)$, il vient

$$
\left|\left(\frac{t^{2}}{\sigma^{2} n}\right)^{-1}\left(\lambda\left(\frac{t}{\sigma \sqrt{n}}\right)-1\right)+\frac{\sigma^{2}}{2}\right| \leqslant \frac{\sigma^{2}}{t^{2}} \varepsilon \leqslant 4 \sigma^{2} \varepsilon .
$$

Soit $u \in \mathbb{R}^{*},|u| \leqslant \frac{1}{\sigma \sqrt{N(\varepsilon)}}$. Il existe clairement $n \geqslant N(\varepsilon)$ tel que $\frac{1}{2 \sigma \sqrt{n}} \leqslant|u| \leqslant \frac{1}{\sigma \sqrt{n}}$. L'inégalité précédente appliquée avec $t=\sigma \sqrt{n} u$ montre que $\left|\frac{\lambda(u)-1}{u^{2}}+\frac{\sigma^{2}}{2}\right| \leqslant 4 \sigma^{2} \varepsilon$.

On suppose maintenant que les conditions des Théorèmes 2.1 ou 2.1' sont satisfaites. Grâce au Lemme 4.1, le théorème (K-L) et le Lemme 4.2 s'appliquent, et l'on est alors en mesure d'utiliser les techniques usuelles de transformée de Fourier pour établir (TLC) et (TLL). À cet effet on pourra, ou bien adapter les preuves classiques des théorèmes limite central ou local pour les suites de v.a.i.i.d. (voir par exemple [4]), ou bien recopier les preuves de [12] (celle du Lemme IV.8 pour (TLC) et celle du Lemme IV.4 pour (TLL)).

Dans les deux cas, il sera commode de définir, pour tous $f \in \mathcal{B}$ et $t \in J$, l'expression $L(t) f=\langle\phi(t), f\rangle v(t)-$ $\langle v, f\rangle \mathbf{1},{ }^{16}$ et grâce à (2), d'écrire lorsque $\mu_{0} \in \mathcal{B}^{\prime}$

$$
\mathbb{E}_{\mu_{0}}\left[f\left(X_{n}\right) \mathrm{e}^{i t S_{n}}\right]=\lambda(t)^{n} v(f)+\lambda(t)^{n}\left\langle\mu_{0}, L(t) f\right\rangle+\left\langle\mu_{0}, N(t)^{n} f\right\rangle .
$$

Preuve des Théorèmes 2.1 et $\mathbf{2 . 1 ^ { \prime }}$, cas stationnaire. On utilisera ici la formule (3) avec $\mu_{0}=v$ et $f \in \mathcal{B}, f \geqslant 0$, quelconque, et l'on observera, d'une part que le théorème (K-L) et le Lemme 4.2 fournissent les propriétés requises pour $\left\langle v, N(t)^{n} f\right\rangle$ et $\lambda(t)^{n},{ }^{17}$ d'autre part que $|\langle v, L(t) f\rangle| \leqslant \vartheta(t)\|f\|$ (Th. (K-L)), $\operatorname{donc} \lim _{t \rightarrow 0}\langle v, L(t) f\rangle=0$.

Preuve des Théorèmes 2.1 et $\mathbf{2 . 1 ^ { \prime }}$, cas non stationnaire. Soit $\mu_{0}$ une probabilité quelconque sur $E$ telle que $\mu_{0} \in \widetilde{\mathcal{B}}^{\prime}$ et soit $f \in \mathcal{B}, f \geqslant 0$. Par hypothèse $\mathcal{B}$ s'envoie continûment dans $\widetilde{\mathcal{B}}$, donc $\mu_{0} \in \mathcal{B}^{\prime}$. Comme précédemment, pour établir (TLC) et (TLL), on pourra adapter [4] ou bien recopier les preuves de [12] (Lemmes IV.8 et IV.4) en utilisant la formule (3) : les termes $\left\langle\mu_{0}, N(t)^{n} f\right\rangle$ et $\lambda(t)^{n}$ sont contrôlés comme dans le cas stationnaire. Pour l'étude du terme $\left\langle\mu_{0}, L(t) f\right\rangle$, on dispose du résultat suivant.

Lemme 4.3. Sous les conditions $(\tilde{\mathcal{F}})$ ou $\left(\widetilde{\mathcal{F}}^{\prime}\right)$, v(t) converge vers 1 dans $\widetilde{\mathcal{B}}$ quand $t \rightarrow 0$.

De ce lemme il résulte que $\left|\left\langle\mu_{0}, L(t) f\right\rangle\right| \leqslant \vartheta(t)\|f\|+C\left\|\mu_{0}\right\|_{\sim}\|v(t)-1\|_{\sim}\|f\|$, où $\|\cdot\|_{\sim}$ désigne la norme $\operatorname{sur} \widetilde{\mathcal{B}}$ et $\widetilde{\mathcal{B}}^{\prime}$. Donc $\lim _{t \rightarrow 0}\left\langle\mu_{0}, L(t) f\right\rangle=0$.

\footnotetext{
${ }^{16}$ Le terme $L(t) f$, noté $L_{n}(t) f$ dans [12], est dans ce travail indépendant de $n$, car d'après (F2), 1 est l'unique valeur propre périphérique de $Q$.

17 D'après $(\beta)$ (Lemme 4.2), on a, pour $|u|$ petit, $|\lambda(u)| \leqslant 1$, et $\left|\lambda\left(\frac{u}{\sigma}\right)\right| \leqslant 1-\frac{u^{2}}{2}+\frac{u^{2}}{4} \leqslant \mathrm{e}^{-u^{2} / 4}$ : la 1ère inégalité suffit dans la preuve de (TLC), la seconde est essentielle dans celle de (TLL).
} 
Preuve du lemme. Nous reprenons ici des arguments déjà utilisés dans [13]. Soit $\Gamma_{1}$ un cercle centré en $\lambda=1$, de rayon $\rho$, avec $\rho$ assez petit pour que, pour $z \in \Gamma_{1}$ et $t \in J$, d'une part $(z-Q)^{-1}$ existe dans $\mathcal{L}(\mathcal{B})$ et $\mathcal{L}(\widetilde{\mathcal{B}})$, d'autre part $(z-Q(t))^{-1}$ existe dans $\mathcal{L}(\mathcal{B})$. Ceci est possible d'après la condition (F2) vérifiée par hypothèse sur $\mathcal{B}$ et $\widetilde{\mathcal{B}}$, et d'après le théorème (K-L). On a pour $f \in \mathcal{B}, z \in \Gamma_{1}$, et $t \in J$,

$$
(z-Q(t))^{-1} f-(z-Q)^{-1} f=(z-Q)^{-1}(Q(t)-Q)(z-Q(t))^{-1} f .
$$

Soit $\widetilde{M}=\sup \left\{\left\|(z-Q)^{-1}\right\|_{\sim}, z \in \Gamma_{1}\right\}$, où $\|\cdot\|_{\sim}$ désigne la norme sur $\mathcal{L}(\widetilde{\mathcal{B}})$; on a $\widetilde{M}<+\infty$ car $Q$ vérifie (F2) sur $\widetilde{\mathcal{B}}$. Soit $\varepsilon(t)$ la norme de $Q(t)-Q$ dans $\widetilde{L}(\mathcal{B}, \widetilde{\mathcal{B}}) ; \varepsilon(t)$ tend vers 0 avec $t$ par hypothèse. Enfin soit $M=$ $\sup \left\{\left\|(z-Q(t))^{-1}\right\|, t \in J, z \in \Gamma_{1}\right\} ;$ on a $M<+\infty$ d'après [16]. D'où

$$
\left\|(z-Q(t))^{-1} f-(z-Q)^{-1} f\right\|_{\sim} \leqslant \tilde{M} \varepsilon(t) M\|f\| .
$$

Le projecteur $\Pi(t)$ définissant $v(t)$ (cf. $\S 4.1$ ) est obtenu dans [16], comme dans la théorie classique, comme l'intégrale curviligne de la résolvante $(z-Q(t))^{-1}$ sur le cercle orienté $\Gamma_{1}$. En intégrant $(z-Q(t))^{-1}-(z-Q)^{-1}$, vue comme application à valeurs dans $\mathcal{L}(\mathcal{B}, \widetilde{\mathcal{B}})$, on déduit de ce qui précède que $\Pi(t) \mathbf{1}$ converge dans $\widetilde{\mathcal{B}}$ vers $\Pi(0) \mathbf{1}=\mathbf{1}$. Le lemme en résulte.

\section{Remerciements}

Les conseils de Jean-Pierre Conze et Hubert Hennion, qui ont accepté de lire la version préliminaire de ce travail, et d'autre part les nombreuses remarques et recommandations du rapporteur, m'ont permis d'améliorer la présentaion de cet article et d'en clarifier certaines parties. Je les en remercie.

\section{Références}

[1] R.B. Bapat, T.E.S. Raghavan, Non-Negative Matrices and Applications, Encyclopedia of Mathematics and its Applications, vol. $64,1997$.

[2] M. Benda, A central limit theorem for contractive stochastic dynamical systems, J. Appl. Probab. 35 (1998) 200-205.

[3] P. Billingsley, The Lindeberg-Levy theorem for martingales, Proc. Amer. Math. Soc. 12 (1961) 788-792.

[4] L. Breiman, Probability, Classic in Applied Mathematics, SIAM, 1993.

[5] A. Broise, F. Dal'bo, M. Peigné, Études spectrales d'opérateurs de transfert et applications, Astérisque 238 (1996).

[6] M. Duflo, Random Iterative Models, Applications of Mathematics, Springer-Verlag, Berlin, Heidelberg, 1997.

[7] Y. Guivarc'h, J. Hardy, Théorèmes limites pour une classe de chaînes de Markov et applications aux difféomorphismes d'Anosov, Ann. Inst. Henri Poincaré 24 (1) (1988) 73-98.

[8] M.I. Gordin, On the central limit theorem for stationary processes, Soviet Math. Dokl. 10 (5) (1969) 1174-1176.

[9] M.I. Gordin, B.A. Lifsic, On the central limit theorem for stationary Markov processes, Soviet Math. Dokl. 19 (2) (1978) $392-394$.

[10] H. Hennion, Sur un théorème spectral et son application aux noyaux lipchitziens, Proc. Amer. Math. Soc. 118 (2) (1993) 627-634.

[11] H. Hennion, Limit theorems for products of positive random matrices, Ann. Probab. 25 (4) (1997) 1545-1587.

[12] H. Hennion, L. Hervé, Limit Theorems for Markov Chains and Stochastic Properties of Dynamical Systems by Quasi-compactness, Lecture Notes in Mathematics, vol. 1766, Springer, Berlin, 2001.

[13] H. Hennion, L. Hervé, Central limit theorems for iterated random lipschitz mappings, Ann. Probab. (2004), sous presse.

[14] I.A. Ibragimov, A central limit theorem for a class of dependant random variables, Theory Probab. Appl. 8 (1963) 83-89.

[15] C.T. Ionescu-Tulcea, G. Marinescu, Théorème ergodique pour des classes d'opérations non complètement continues, Ann. of Math. 52 (1) (1950) $140-147$.

[16] G. Keller, C. Liverani, Stability of the spectrum for transfer operators, Ann. Scuola Norm. Sup. Pisa. CI. Sci. (4) XXVIII (1999) $141-152$.

[17] A.N. Kolmogorov, A local limit theorem for classical Markov chains, Izv. Akad. Nauk. SSSR. Ser. Math. 13 (1949) $281-300$.

[18] S.P. Meyn, R.L. Tweedie, Markov Chains and Stochastic Stability, Springer Verlag, Berlin, 1993.

[19] X. Milhaud, A. Raugi, Etude de l'estimateur du maximum de vraisemblance dans le cas d'un processus auto-régressif : convergence, normalité asymptotique, vitesse de convergence, Ann. Inst. H. Poincaré 25 (4) (1989) 383-428.

[20] S.V. Nagaev, Some limit theorems for stationary Markov chains, Theory Probab. Appl. 11 (4) (1957) 378-406.

[21] S.V. Nagaev, More exact statements of limit theorems for homogeneous Markov chains, Theory Probab. Appl. 6 (1) (1961) 62-81.

[22] R.D. Nussbaum, The radius of essential spectrum, Duke Math. J. 37 (1970) 473-478. 
[23] B. Petit, $\theta$-transformations, $\theta$-shifts an limit theorems for Riesz-Raikov sums, Ergodic Theory Dynam. Systems 16 (1996) 335-364.

[24] J. Rousseau-Egele, Un théorème de la limite locale pour une classe de transformations dilatantes, Ann. Probab. 11 (3) (1983) 772-788.

[25] H. Schaeffer, Banach Lattices and Positive Operators, Springer-Verlag, Berlin, Heidelberg, New York, 1974.

[26] M. Seva, On the local limit theorem for non-uniformly ergodic Markov chains, J. Appl. Probab. 32 (1995) 52-62. 